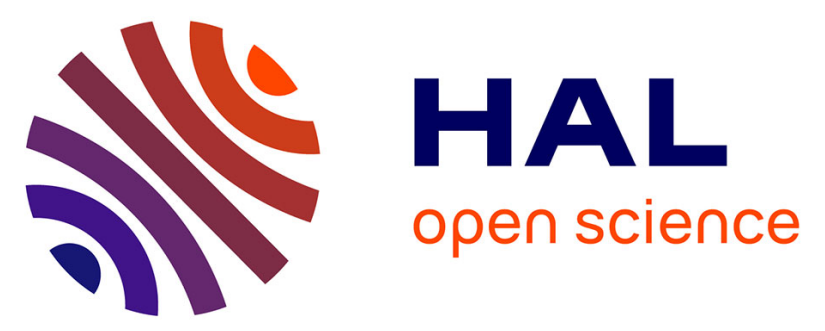

\title{
A method for measuring the acoustic properties of a porous sample mounted in a rigid ring in acoustic tubes
}

Thomas Dupont, Philippe Leclaire, Raymond Panneton, Kévin Verdière, Saïd Elkoun

\section{- To cite this version:}

Thomas Dupont, Philippe Leclaire, Raymond Panneton, Kévin Verdière, Saïd Elkoun. A method for measuring the acoustic properties of a porous sample mounted in a rigid ring in acoustic tubes. 21st International Congress on Acoustics (ICA 2013), Acoustical Society of America, Jun 2013, Montréal, Canada. pp.065008, 10.1121/1.4799701 . hal-01329863

\section{HAL Id: hal-01329863 https://hal.science/hal-01329863}

Submitted on 9 Jun 2016

HAL is a multi-disciplinary open access archive for the deposit and dissemination of scientific research documents, whether they are published or not. The documents may come from teaching and research institutions in France or abroad, or from public or private research centers.
L'archive ouverte pluridisciplinaire HAL, est destinée au dépôt et à la diffusion de documents scientifiques de niveau recherche, publiés ou non, émanant des établissements d'enseignement et de recherche français ou étrangers, des laboratoires publics ou privés.

\section{(c)(1)}

Distributed under a Creative Commons Attribution| 4.0 International License 


\title{
Proceedings of Meetings on Acoustics
}

\author{
ICA 2013 Montreal \\ Montreal, Canada 2 - 7
}

June 2013

\section{1aSA8. A method for measuring the acoustic properties of a porous sample mounted in a rigid ring in acoustic tubes}

\author{
Thomas Dupont*, Philippe Leclaire, Raymond Panneton, Kévin Verdière and Saïd Elkoun
}

* Corresponding author's address: Drive, ISAT, Université de Bourgogne, 49 Rue Mademoiselle Bourgeois, Nevers, 58027,

\section{Bourgogne, France, thomas.dupont@u-bourgogne.fr}

This study presents a method to measure the acoustic properties of a homogeneous porous material with a support or a reduction element in an acoustic tube. Some materials tested have a lateral size much smaller than the tube's diameter, as they cannot be produced in the correct dimensions without corrupting the material; this also permits the testing of the same samples in a large frequency bandwidth by using different section tubes. Moreover, the acoustic leaks on the material boundaries can significantly change the transmission loss measured in tubes. To rectify these problems, rings can be placed on each material surface. The presence of these rings can influence the acoustic indicator measurement; while this effect is negligible for tubes with a large cross section, it is not for tubes with a small cross section. To correct, or remove, the influence of the rings, we propose to use an application of the parallel assembly process of the transfer matrix method which has recently been proposed by Panneton et al. [Proceeding Internoise New York (2012)]. Measurements on classical porous materials with and without reductions are proposed and compared to simulated results. The ring's effects and the proposed corrections are discussed for different materials. 


\section{INTRODUCTION}

Measurements in an acoustic tube are currently used to characterize the acoustic properties of a material. ${ }^{1}$ The acoustic indicators (sound absorption and transmission) can be measured by the methods proposed in refs. 2 and 3 with normal plane wave excitation assumption. These methods were developed to characterize materials for which the tested sample has a homogeneous surface that perfectly fits the tube's cross section. Nevertheless, some material can have non-homogeneous surface; for example, the systems can be made up of different materials which are stacked in parallel. ${ }^{4}$ Moreover, some materials tested in practical applications sometimes have a lateral size smaller than the tube diameter, as they cannot be produced in the correct dimensions; samples of smaller sizes are also used in smaller impedance tubes for testing in a higher frequency range. In these cases an adaptor can be used (see Figure 1). However, even if the sample diameter is correct, the acoustic leaks on the material boundaries can significantly change the transmission loss measured in tubes. To correct these problems, rings can be placed on each material surface (see Figure 1). Both presence of these rings and the diameter adaptor can individually influence the acoustic indicator measurements. While this effect can be neglected for tubes with a large cross section and small rings or adaptor, it cannot for larger rings or adaptors.

Recently, the authors have presented an extension of the transfer matrix method to deal with parallel assembly of transfer matrices. ${ }^{4}$ This parallel assembly allows the study of acoustic materials which are in the form of mosaics or patchworks. In this paper, it is proposed to use this method to define the acoustic indicators of a material when it is tested with a support (or a ring). To apply this method, it is necessary to know the support material's acoustic parameters (JCA parameters). In this paper, the theory is presented in the first part, and comparisons between present model and finite element simulations are presented for a complex composed of two different materials in the second part. The application of the approach on an unknown material tested with a rigid and impervious ring is presented in the third part. Comparison between the present approach and experimental results are presented. A correction to take into account a possible end effect is discussed in the last part.

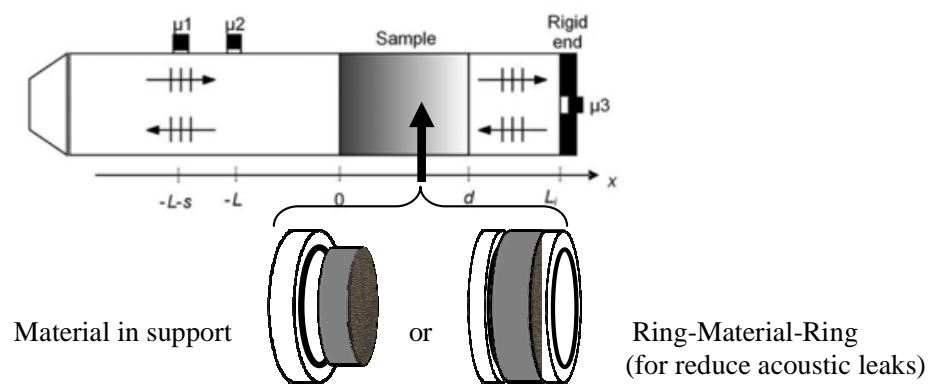

FIGURE 1. Example of acoustic tube ${ }^{3}$ with a material and its support (in the case where the sample lateral size is smaller than the tube diameter) or with two rings (to diminish the acoustic leaks on the sample boundaries).

\section{THEORY}

\section{Presentation of the approach}

This part summarizes the development of the approach and gives principal results; details on the assumptions and the approach are given in references 4 and 5.

As it is presented by K. Verdière in another paper for ICA Montreal, ${ }^{5}$ the transfer Matrix Method (TMM) is a method to predict sound absorption and sound transmission loss of multilayered laterally infinite materials stacked in series. ${ }^{1}$ As it is noted in ref 5 for a parallel arrangement of $N$ elements, the transfer matrix for each element is given by: 


$$
\left\{\begin{array}{l}
P\left(M_{i}\right) \\
U\left(M_{i}\right)
\end{array}\right\}=\mathbf{T}_{i}\left\{\begin{array}{l}
P\left(M_{i}^{\prime}\right) \\
U\left(M_{i}^{\prime}\right)
\end{array}\right\}=\left[\begin{array}{ll}
t_{i, 11} & t_{i, 12} \\
t_{i, 21} & t_{i, 22}
\end{array}\right]\left\{\begin{array}{l}
P\left(M_{i}^{\prime}\right) \\
U\left(M_{i}^{\prime}\right)
\end{array}\right\}
$$

where $P$ is acoustic pressures, $U$ is the $x$-component the acoustic velocities and $t_{i, m n}$ are the coefficients of transfer matrix $\mathbf{T}_{i}$. To express the global transfer matrix $\left[\mathrm{T}_{\mathrm{p}}\right]$ it is proposed to use the admittance matrix of each element. This matrix are given by:

$$
\left\{\begin{array}{l}
U\left(M_{i}\right) \\
U\left(M_{i}^{\prime}\right)
\end{array}\right\}=\left[\begin{array}{ll}
y_{i, 11} & y_{i, 12} \\
y_{i, 21} & y_{i, 22}
\end{array}\right]\left\{\begin{array}{l}
P\left(M_{i}\right) \\
P\left(M_{i}^{\prime}\right)
\end{array}\right\}=\frac{1}{t_{i, 12}}\left[\begin{array}{cc}
t_{i, 22} & t_{i, 21} t_{i, 12}-t_{i, 22} t_{i, 11} \\
1 & -t_{i, 11}
\end{array}\right]\left\{\begin{array}{l}
P\left(M_{i}\right) \\
P\left(M_{i}^{\prime}\right)
\end{array}\right\} .
$$

Consider now the continuity of flow rate and pressure at the open interface for each element:

$$
\left\{\begin{array}{l}
P\left(M_{i}\right) \\
P\left(M_{i}^{\prime}\right)
\end{array}\right\}=\left\{\begin{array}{c}
P \\
P^{\prime}
\end{array}\right\} \text { and }\left\{\begin{array}{c}
U \\
U^{\prime}
\end{array}\right\}=\left\{\begin{array}{c}
\sum r_{i} U\left(M_{i}\right) \\
\sum r_{j} U\left(M_{j}^{\prime}\right)
\end{array}\right\} \text {. }
$$

with $r_{i}$ is the surface ratio of element $i$ given by $S_{i} / S_{\text {total }}$ where $S_{i}$ and $S_{\text {total }}$ are the cross-sectional surface areas of element $i$ and the construction, respectively.

The transfer matrix $\left[\mathbf{T}_{\mathbf{P}}\right]$ of the parallel construction is given by:

$$
\left\{\begin{array}{l}
P \\
U
\end{array}\right\}=\mathbf{T}_{\mathbf{p}}\left\{\begin{array}{l}
P^{\prime} \\
U^{\prime}
\end{array}\right\}=\frac{-1}{\Sigma r_{i} y_{i, 21}}\left(\begin{array}{cc}
\Sigma r_{i} y_{i, 22} \Sigma r_{i} y_{i, 11}-\Sigma r_{i} y_{i, 12} \Sigma r_{i} y_{i, 21} & -\Sigma r_{i} y_{i, 11}
\end{array}\right)\left\{\begin{array}{l}
P^{\prime} \\
U^{\prime}
\end{array}\right\} .
$$

\section{Application to the acoustic of a unknown material tested with a support or rings}

We propose to use the approach in a "reverse way" to determine the acoustic indicator of one unknown element. We propose to use the transfer matrix of the complex (measured or simulated) and the Johnson-Champoux-Allard (JCA) parameters of the other elements to determine the transfer matrix of the unknown element.

Consider now a complex composed by two elements in parallel construction (see figure 1), an unknown porous or fibrous material and a support material for which JCA parameters are known. We suppose that the unknown material and support material are homogeneous with rigid frame and they respect the JCA assumptions1. The subscript $p, s$ and $m$ represent respectively the complex, the support and the unknown material. The complex material is tested in an acoustic tube and all the components of the transfer matrix $\left[T_{p}\right]$ are measured or defined.

The support material matrix $\left[T_{s}\right]$ is given by:

$$
\left[T_{s}\right]=\left[\begin{array}{lc}
\cos \left(k_{s} l\right) & j Z_{s} \sin \left(k_{s} l\right) \\
\frac{j}{Z_{s}} \sin \left(k_{s} l\right) & \cos \left(k_{s} l\right)
\end{array}\right],
$$

where $l$ is the complex thickness, $k_{S}$ and $Z_{S}$ are the equivalent wave number and acoustic impedance of the support material, calculated with the Johnson-Champoux-Allard (JCA) equivalent fluid approach with rigid frame.

The admittance matrix of the unknown element $\left[Y_{m}\right]$ is given by:

$$
\left[Y_{m}\right]=\frac{1}{r_{m} t_{p, 12}}\left[\begin{array}{cc}
t_{p, 22}-r_{s} y_{s, 11} t_{p, 12} & -\operatorname{det}\left[T_{p}\right\rfloor-r_{s} y_{s, 12} t_{p, 12} \\
1-r_{s} y_{s, 21} t_{p, 12} & -t_{p, 11}-r_{s} y_{s, 22} t_{p, 12}
\end{array}\right],
$$

where det $\left[T_{p}\right]$ represents the determinant of $\left[T_{p}\right]$, and $y_{s, i j}$ are the component of the admittance matrix of support material calculated with the help of (2). 
The transfer matrix of the unknown element $\left[T_{m}\right]$ is given by:

$$
\left[T_{m}\right]=\frac{1}{1-t_{p, 12} r_{s} y_{s, 21}}\left[\begin{array}{cc}
t_{p, 11}+r_{s} y_{s, 22} t_{p, 12} & r_{m} t_{p, 12} \\
-\operatorname{det}\left(Y_{m}\right) r_{m} t_{p, 12} & t_{p, 22}+r_{s} y_{s, 11} t_{p, 12}
\end{array}\right]
$$

with

$$
\operatorname{det}\left[Y_{m}\right]=\frac{1}{\left(r_{m} t_{p, 12}\right)^{2}}\left[-\left(t_{p, 22}-r_{s} y_{s, 11} t_{p, 12}\right)\left(t_{p, 11}+r_{s} y_{s, 22} t_{p, 12}\right)+\left(1-r_{s} y_{s, 21} t_{p, 12}\right)\left(\operatorname{det}\left[T_{p}\right]+r_{s} y_{s, 12} t_{p, 12}\right)\right] .
$$

From the transfer matrix of the unknown material, normal absorption coefficient and normal sound transmission loss of the unknown material can be calculated by, respectively:

$$
\alpha=1-\left|\frac{t_{m, 11}-t_{m, 21} Z_{0}}{t_{m, 11}+t_{m, 21} Z_{0}}\right|^{2} \text { and } n S T L=20 \log \left(\frac{1}{2}\left|t_{m, 11}+t_{m, 22}+\frac{t_{m, 12}}{Z_{0}}+t_{m, 21} Z_{0}\right|\right)
$$

\section{VALIDATION OF THE APPROACH}

A comparison between the present approach and virtual measurements obtained with a 3D acoustical FEM simulations using COMSOL software is proposed. A three-microphone method is used to get the virtual measurements (see figure 1).3 In the FEM model on COMSOL, parabolic tetrahedral elements were used to mesh the different acoustic domains of the tube, and convergence of the results was verified in the frequency range of interest. The elements of the construction samples were modeled as equivalent fluids using complex sound speed $\left(c_{e q}=\omega / k_{e q}\right.$, where $\omega$ is the angular frequency) and dynamic density $\left(\rho_{\mathrm{eq}}=Z_{c e q} k_{e q} / \omega\right)$ given by the JCA model. Plane wave in normal incidence is chosen for the acoustic excitation. Another configuration is proposed to validate the present model: a cylinder $20 \mathrm{~mm}$ thick with an $80 \mathrm{~mm}$ section diameter of unknown material (felt) is coupled with a ring support of melamine foam (100-80 mm outer and inner diameters), and the assembly is placed in a threemicrophone acoustic tube with a diameter of $100 \mathrm{~mm}$ (see figure 1). Table 1 gives the JCA parameters of the two elements in the assembly. Based on the virtual measurement (FEM) of the transfer matrix of the assembly, the transfer matrix of the unknown material is deduced with the present model. The normal sound absorption coefficient is calculated for the unknown material, and the unknown material was tested alone with a tube that has the same diameter. Note that in this case, the cutoff frequency of the tube has to be chosen for the configuration with the largest diameter tube.

Comparisons between the normal sound absorption coefficient found with FEM virtual measurement and that found with the present model are given in figure 2. The present model and the FEM virtual measurements are in excellent agreement. Consequently, the objective of finding the curve of the material alone (dots) from the curve

\begin{tabular}{|c|c|c|c|c|c|c|c|}
\hline Materials & $\begin{array}{c}\text { Open } \\
\text { porosity }\end{array}$ & $\begin{array}{c}\text { Static } \\
\text { airflow } \\
\text { resistivity } \\
\left(\text { N.s.m }{ }^{-4}\right)\end{array}$ & $\begin{array}{c}\text { Viscous } \\
\text { characteristic } \\
\text { length }(\mu \mathrm{m})\end{array}$ & $\begin{array}{c}\text { Thermal } \\
\text { characteristic } \\
\text { length }(\mu \mathrm{m})\end{array}$ & Tortuosity & $\begin{array}{c}\text { Surface } \\
\text { ratio of } \\
\text { element } \\
\mathbf{r}_{\mathbf{i}} \\
\end{array}$ & $\begin{array}{c}\text { Thickness } \\
\text { of element } \\
\text { (mm) }\end{array}$ \\
\hline $\begin{array}{l}\text { Annular ring } \\
\text { adaptor : } \\
\text { Melamine foam }\end{array}$ & 0.98 & 10000 & 100 & 300 & 1.02 & 0.36 & 20 \\
\hline $\begin{array}{l}\text { Unknown material } \\
\text { Felt }\end{array}$ & 0.98 & 26000 & 49 & 98 & 1.00 & 0.64 & 20 \\
\hline
\end{tabular}
of the material in the annular support (red curve) from the proposed method is achieved.

TABLE 1. JCA parameters of element materials. 


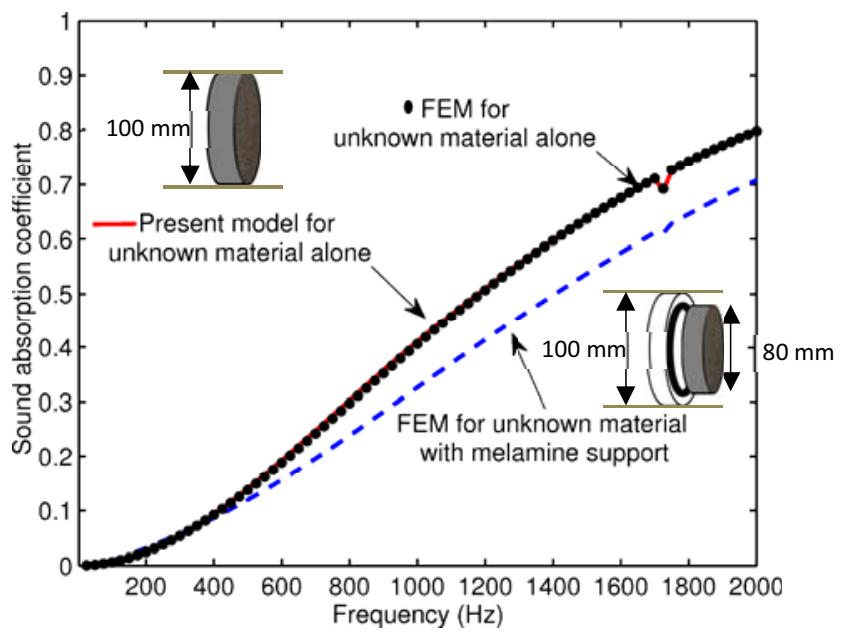

FIGURE 2. Virtual measurements (FEM and present model) of the normal sound absorption coefficient for two configurations. Conf.1 : cylinder with an $80 \mathrm{~mm}$ section diameter of unknown material (felt) coupled with a ring support (melamine foam) placed in an acoustic tube of $100 \mathrm{~mm}$ section diameter. Conf. 2 : cylinder of $100 \mathrm{~mm}$ diameter of unknown material (felt) placed alone in the acoustic tube of $100 \mathrm{~mm}$ section diameter.

\section{APPLICATION TO MEASUREMENTS OF MATERIALS WITH RIGID ANNULAR ADAPTOR}

\section{Proposed approach}

Now consider infinitely rigid and impervious annular adaptor (or rings for acoustics leaks). The adaptor represents in this case a tube diameter reduction. The impedance of the annular ring material tends to infinity and the transfer matrix of unknown material (6) becomes:

$$
\left[T_{m}\right]_{\text {rigid_sup }}=\left[\begin{array}{cc}
t_{p, 11} & t_{p, 12} r_{m} \\
t_{p, 21} / r_{m} & t_{p, 22}
\end{array}\right] .
$$

The same transfer matrix could be found if the continuities of flow rate and pressure are directly included in the transfer matrix expression (1):

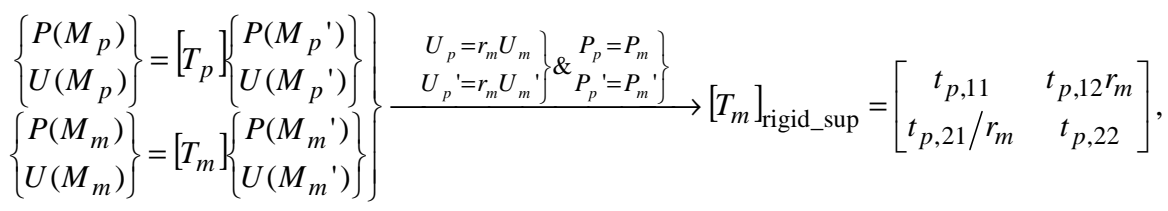

where $M_{p}$ and $M_{p}$ ' are points "just outside" the reduction, and $M_{m}$ and $M_{m}$ ' are points "just inside" the reduction (just in front of and behind the unknown material).

It can be noted that if only sound absorption is considered, the surface impedance of the assembly coupled with rigid piston without an air cavity is given by: 


$$
Z_{p}^{s}=\frac{t_{p, 11}}{t_{p, 21}}
$$

and the surface impedance of the unknown material coupled with a rigid piston in a tube with the same diameter is given by (with (10)):

$$
Z_{m}^{s}=\frac{t_{m, 11}}{t_{m, 21}}=\frac{t_{p, 11}}{t_{p, 21}} r_{m}=Z_{p}^{s} r_{m}
$$

The normal sound absorption coefficient is given by :

$$
\alpha=1-\left|\frac{Z_{m}^{s}-Z_{0}}{Z_{m}^{s}+Z_{0}}\right|^{2} .
$$

The same result is found when the assembly is coupled with an air cavity and a rigid termination. The surface impedance of the unknown material coupled with an air cavity and rigid termination is given by:

$$
Z_{m+\text { aircavity }}^{s}=Z_{p+\text { aircavity }}^{s} r_{m}
$$

To conclude this section, if only sound absorption is considered in the case of a material coupled with a rigid and Impervious annular ring adaptor, it is not necessary to measure the complex transfer matrix; the surface impedance of assemblies with or without air cavity measurements are enough to determine the sound absorption coefficient of the unknown material with or without air cavity in an adapted tube.

\section{Validation section}

Comparisons between the absorption coefficient (measured or found with eq. (15)) between assemblies (unknown material with a rigid annular ring adaptor) and unknown material with correct diameter coupled with air cavity and rigid piston are proposed. A two-microphone impedance tube is used to measure the normal sound absorption coefficient of the materials coupled to an air cavity and a rigid termination. The tube diameter is $100 \mathrm{~mm}$. The two microphones upstream the sample are used to measure the sound absorption by the standard impedance tube measurement technique. 2 Two samples are tested: a $31 \mathrm{~mm}$ thick melamine foam sample and a $20 \mathrm{~mm}$ thick sample of the same material. The JCA parameters are given in Table 1. The samples that are tested with the annular ring adaptor are cylinders with a $80 \mathrm{~mm}$ section diameter. The rigid ring has outer and inner diameters of $100 \mathrm{~mm}$ and $80 \mathrm{~mm}$, respectively. The surface impedance of the unknown material is found with the help of the surface impedance of the assembly involving the ring adaptor and measured in the tube. A measurement of the acoustic absorption coefficient of a sample of the same material but without diameter adaptation is done for the comparisons. In this case the sample is a $100 \mathrm{~mm}$ diameter cylinder. 

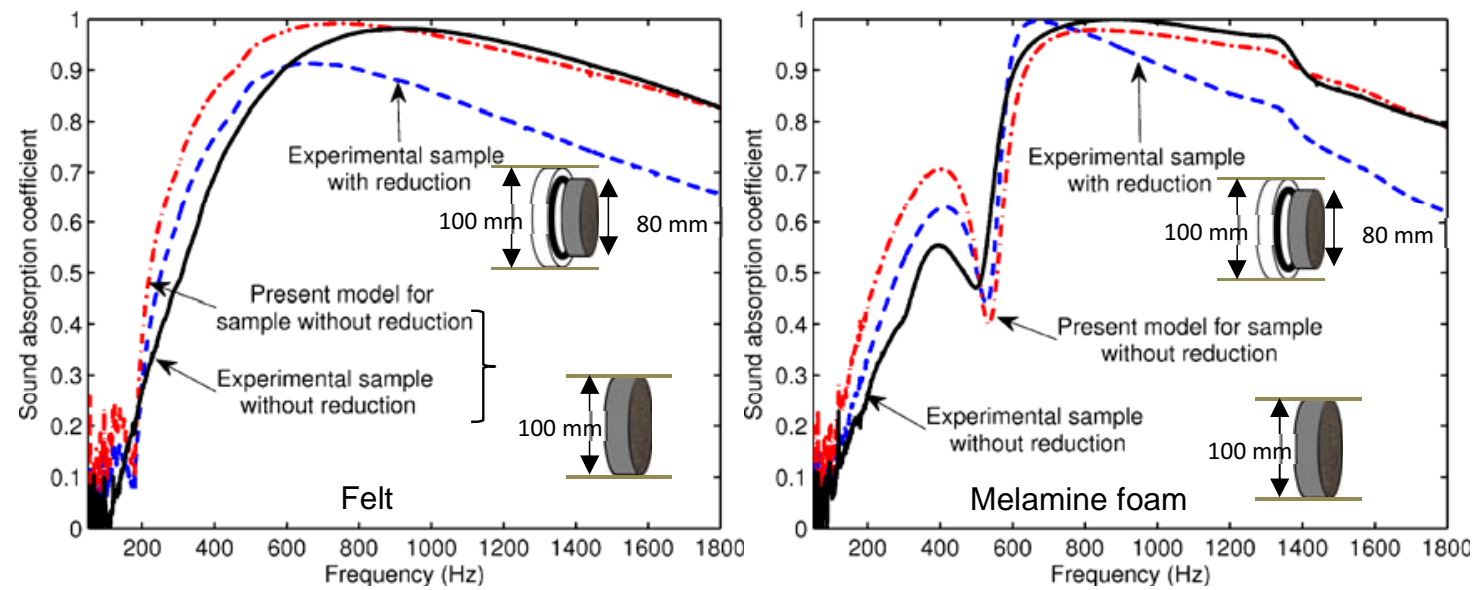

FIGURE 3. Experimental measurements (FEM and present model) of normal sound absorption coefficient for two configurations and two different samples (left, felt; right, melamine foam). Conf. 1: cylinder with an $80 \mathrm{~mm}$ section diameter of unknown material (felt) coupled with a ring adaptor placed in an acoustic tube of $100 \mathrm{~mm}$ diameter, followed by a $50 \mathrm{~mm}$ air cavity and a rigid termination. Conf. 2: cylinder with a $100 \mathrm{~mm}$ section diameter of unknown material (felt) placed alone the acoustic tube of $100 \mathrm{~mm}$ section diameter.

For the melamine foam sample, a good agreement is obtained between the absorption coefficient for the sample without ring adaptor measured and found with the present approach. Difference can be noted in the frequency range around a structural resonance peak.

For the felt sample the comparison between the absorption coefficients is good after the first absorption peak. It can be noted that the absorption peak frequency is higher for the measured results than the present approach results. A possible interpretation comes from the fact that for this material, the radiation end effect of rigid annular ring adaptor could appear and could modify the acoustic behavior of the complex. The present approach is based on the sample with annular ring adaptor measurements but the "reverse" process doesn't take into account the end effect. This end effect has no meaning for samples tested without ring adaptors. So if in measurements this effect occurs, it will change the prediction of the acoustic behavior of the sample in the tube. In the next part of the paper, a discussion is proposed on this point. Another possible interpretation comes from the fact for an heterogeneous (in section) material, the acoustic wave in the air cavity is not plane for small cavity, in this case the air cavity representation by transfer matrix is not totally right. Indeed in certain cases, (small) difference can be noted between the transfer matrix approach and the FEM approach. Samples with ring adaptor and with more depth air cavity have to be tested.

\section{Radiation end effect discussions}

If the ring is infinitely rigid and impermeable and if the unknown material is sufficiently permeable, an end termination effect could appear (also called diaphragm effect). This effect has as consequence an added inertial effect and modified acoustic indicators (indeed the absorption peaks frequencies decrease with this effect). To take into account a possible end effect, it is proposed here to use the classical approach of the sound radiation of a rigid piston in infinitely rigid baffle proposed by Rayleigh.6 However the ring has finite dimensions and the end correction depends on the surface ratio of material $r_{m}$ and on the JCA parameters of the unknown material. The end correction can be evaluated in a first approximation by the empirical Fok approach7 for structure in tube. To express the transfer matrix, we propose to use same approach developed by Auregan8 to take into account a sudden area expansions effect in dissipative silencers. With help of (6), the transfer matrix of the complex expression:

$$
\left[T_{p}\right]_{\text {rigid_sup }}=\left[\begin{array}{cc}
1 & Z_{\text {add }} \\
0 & 1
\end{array}\right]\left[\begin{array}{cc}
t_{m, 11} & t_{m, 12} / r_{m} \\
t_{m, 21} r_{m} & t_{m, 22}
\end{array}\right]\left[\begin{array}{cc}
1 & Z_{\text {add }} \\
0 & 1
\end{array}\right],
$$


with the added impedance (added part for reactance) given by:

$$
Z_{\text {add }}=j \rho_{0} \omega \frac{4}{3 \pi} \frac{d_{m}}{\Psi_{\text {Fok }}\left(r_{m}\right)}
$$

where $\rho_{0}$ is the volume density of air, $\omega$ is the angular frequency, $d_{m}$ the section diameter of the sample and $\Psi_{F o k}\left(r_{m}\right)$ is the Fok function given by:

$$
\Psi_{\text {Fok }}^{-1}\left(r_{m}\right)=\sum_{i=0}^{12} a_{i}\left(\frac{\sqrt{\pi r_{m}}}{2}\right)^{i},
$$

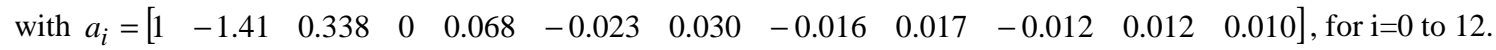

When the Fok function is applied on the last configuration (ring support with an exterior and interior diameter of 100 and $80 \mathrm{~mm}$, respectively), the added impedance becomes insignificant and no change on absorption coefficient is observed. If the Fok function is not used, the added impedance is too large and the absorption coefficient prediction decreases. The next step for this project will be to develop added impedance that is better adapted for the materials studied.

\section{CONCLUSION}

Based on an extension of the transfer matrix method to deal with parallel assembly of transfer matrices, a simple method is proposed to define the acoustic indicators of a material when it is tested with a support (adaptor) or a ring. A general approach was developed and validated for a complex with two different materials (support and unknown material). Based on the matrix transfer measurements of a complex (unknown material and support) and on the JCA parameters of the support, this method allows the determination of the transfer matrix of the material.

An application of measurements of materials with rigid annular adaptor was proposed. The formulation is very simple. For material with rigid and impermeable support, if only sound absorption is considered it is not necessary to measure the complex transfer matrix, the surface impedance of complex with or without air cavity measurements is enough to determine the sound absorption coefficient of the unknown material with or without air cavity in an adapted tube. Attention has to be paid in the choice of air cavity depth when material and annular adaptor is used.

An end effect for rigid ring support can occur. This effect depends on JCA parameters and on the surface ratio of material $r_{\mathrm{m}}$. It has to be studied in more detail and a new development of added impedance should be carried out.

\section{REFERENCES}

1 J.F. Allard and N. Atalla, Propagation of sound in porous media: modeling sound absorbing materials, 2nd ed. (Elsevier Applied Science, New York, 2009), pp. 243-307.

2 ISO-10534-2, "Acoustics-Determination of sound absorption coefficient and impedance in impedance tubes. Part 2: Transfer-function method," International Organization for Standardization, Geneva, Switzerland 1998.

3 Y. Salissou, R. Panneton, and O. Doutres, "Three-microphone method for measuring the normal sound transmission loss of noise control samples in standing wave tube," submitted for publication in Applied Acoustics (2010).

4 K.Verdière, R. Panneton, S. Elkoun, T. Dupont and P. Leclaire, "Transfer matrix method applied to the parallel assembly of sound absorbing materials," J. Acoust. Soc. Am, submitted (2012).

5 K.Verdière, R. Panneton, S. Elkoun, T. Dupont and P. Leclaire, "Prediction of acoustic properties of parallel assemblies by means of transfer matrix method", proceeding ICA Montreal, june 2013.

6 L. Rayleigh. Theory of sound II. Macmillan, New-York, 1929.

7 V.A. Fok. Doklady akademii nauk. SSSR, 31, 1941.

8 Y. Aurégan and A. Debray, "Low frequency sound propagation in a coaxial cylindrical duct: application to sudden area expansions and to dissipative silencers" Journal of Sound and Vibration 243, 461-473 (2001). 